\title{
Acute cannabinoid receptor type 1 (CB1R) modulation influences insulin sensitivity by an effect outside the central nervous system in mice
}

\author{
D. Song • R. H. J. Bandsma $\cdot$ C. Xiao $\cdot$ L. Xi $\cdot$ W. Shao • \\ T. Jin • G. F. Lewis
}

Received: 16 December 2010 / Accepted: 18 January 2011 / Published online: 22 February 2011

(C) Springer-Verlag 2011

\begin{abstract}
Aims/hypothesis Modulation of central nervous system (CNS) and extra-CNS cannabinoid receptor type 1 (CB1R) affects metabolic conditions, independently of weight loss. Here we examined the relative contributions of acute CNS and extra-CNS CB1R modulation on insulin sensitivity using pharmacological gain- and loss-of-function of CB1R in mice.

Methods We assessed the effects of acute modulation of CB1R on insulin sensitivity and tissue glucose uptake by administering a CB1R agonist (HU210) and antagonist (AM251) (vs vehicle) i.v. in wild-type mice. In addition, we administered a CB1R agonist (vs vehicle) systemically (i.v.) to $\mathrm{Cblr}$ (also known as $\mathrm{Cnrl}$ ) knockout $\left(\mathrm{Cblr}^{-/-}\right)$ mice or intracerebroventricularly (i.c.v.) in wild-type mice to elucidate the peripheral vs CNS-mediated regulatory effect of CB1R on insulin sensitivity.
\end{abstract}

D. Song and R. H. J. Bandsma contributed equally to this study.

Electronic supplementary material The online version of this article (doi:10.1007/s00125-011-2082-z) contains supplementary material, which is available to authorised users.

D. Song $\cdot$ R. H. J. Bandsma $\cdot$ C. Xiao $\cdot$ L. Xi $\cdot$ G. F. Lewis

Department of Medicine, University of Toronto,

Toronto, ON, Canada

D. Song • R. H. J. Bandsma • C. Xiao • L. Xi • W. Shao • T. Jin •

G. F. Lewis

Department of Physiology, University of Toronto,

Toronto, ON, Canada

G. F. Lewis $(\bowtie)$

Toronto General Hospital,

200 Elizabeth Street, EN12-218,

Toronto, ON, Canada M5G 2C4

e-mail: gary.lewis@uhn.on.ca
Results HU210 induced significant insulin resistance in wild-type mice with a reduction of whole-body glucose disappearance rate and muscle Akt phosphorylation, as well as of glucose uptake by skeletal muscle, but not by adipose tissue, changes that were prevented by pretreatment with AM251. HU210 did not affect insulin sensitivity in $\mathrm{Cb}_{1} \mathrm{r}^{-/}$ mice, suggesting that the observed effects were mediated through CB1R. HU210 administered i.c.v. did not induce insulin resistance, suggesting that acute stimulation of CNS CB1R was not required for this effect.

Conclusions/interpretation Skeletal muscle insulin sensitivity is affected by acute CB1R modulation. These changes are mediated by extra-CNS CB1R, probably by the receptors in skeletal muscle tissue.

Keywords Cannabinoid receptor type 1 - Glucose disappearance rate - Insulin resistance - Insulin sensitivity . Tissue glucose uptake

$\begin{array}{ll}\text { Abbreviations } \\ \text { CB1R } & \text { Cannabinoid receptor type } 1 \\ \mathrm{Cblr}^{-/} & \text {Whole-body } \mathrm{Cb} 1 r \text { knockout mice } \\ \mathrm{CNS} & \text { Central nervous system } \\ \text { 2-DG } & \text { 2-Deoxy-D- }\left[1-{ }^{14} \mathrm{C}\right] \text { glucose } \\ \text { EGP } & \text { Endogenous glucose production } \\ \text { ERK } & \text { Extracellular signal-regulated protein kinase } \\ \text { Ginf } & \text { Glucose infusion rate } \\ \text { i.c.v. } & \text { Introcerebroventricular } \\ R_{\mathrm{d}} & \text { Glucose disappearance rate }\end{array}$

\section{Introduction}

The prevalence of obesity with its adverse effects on health threatens to undermine recent gains in the prevention of 
atherosclerotic cardiovascular disease, type 2 diabetes and hypertension [1]. Antagonism of the cannabinoid receptor type $1(\mathrm{CB} 1 \mathrm{R})$ of the endocannabinoid system represents a novel pharmacological treatment for obesity and the metabolic syndrome. CB1R antagonists have been shown in clinical trials to have highly beneficial effects, inducing weight loss and improving carbohydrate and lipid metabolism in individuals with obesity and related metabolic disorders [2-4]. However, a major limiting factor of CB1R antagonists that cross the blood-brain barrier is that they are associated with serious central nervous system (CNS) side effects such as depression and anxiety, thereby limiting their potential clinical utility [3].

CB1R is highly abundant in the brain $[5,6]$. Besides the CNS, CB1Rs are also widely expressed in tissues outside the CNS, such as liver, adipose tissue, skeletal muscle and the pancreas [7-11]. There is evidence that several therapeutic benefits of CB1R antagonists are mediated by their interaction with $\mathrm{CB} 1 \mathrm{R}$ in peripheral tissues outside the CNS and that many of their effects are independent of effects on appetite and changes in body weight. For example, in diet-induced obese mice, chronic treatment with rimonabant, a CB1R antagonist, caused a transient reduction in food intake but sustained reduction in body weight [12, 13]. Body weight reductions through $\mathrm{CB} 1 \mathrm{R}$ antagonism can only partially be explained by reductions in food intake; moreover, a variety of metabolic benefits of CB1R antagonism occur independently of reductions in energy intake [7, 12-14], suggesting that peripheral tissue CB1R plays a role in inducing weight loss. In diet-induced obesity, chronic CB1R antagonism causes weight loss and improves insulin sensitivity by diverting lipids from storage toward utilisation, a process that occurs independently of food intake [15]. Whole-body Cblr (also known as $\mathrm{Cnrl})$ knockout $\left(\mathrm{Cblr^{-/- }}\right)$ mice are resistant to dietinduced obesity, while wild-type littermates on the same diet with identical energy intake become obese [16]. In addition, there is direct experimental evidence that CB1R antagonism increases energy expenditure in peripheral tissues $[14,17,18]$.

Recent studies suggest that the endocannabinoid system is involved in modulating carbohydrate metabolism and insulin sensitivity. Chronic antagonism of CB1R leads to weight loss and improved insulin sensitivity in animal models of obesity and in humans [2, 4, 19-21]. However, it is still not clear whether the beneficial effects on insulin sensitivity and metabolic profiles are secondary to reduction in food intake with consequent weight loss or caused by other unrelated mechanisms. Moreover, the tissue sites and contribution of extra-CNS CB1R modulation to wholebody insulin sensitivity, while generally appreciated to play a major role in the metabolic effects of CB1R modulation, are also not well understood.

In the present study, we assessed the effects of acute modulation of CB1R on whole-body insulin sensitivity and tissue glucose uptake, and elucidated the role of the different tissue sites in regulating insulin sensitivity.

\section{Methods}

Animals Male wild-type C57BL/6 mice were obtained from Charles River Laboratories (Wilmington, MA, USA) at the age of 6 to 7 weeks. $\mathrm{Cb}^{-/-}$mice were kindly provided by G. Kunos at the National Institutes of Health (Bethesda, MD, USA). $\mathrm{Cblr}^{-/-}$and $\mathrm{Cb}^{+/+}$(littermate) mice were obtained by breeding of heterozygotes that had been backcrossed to a C57BL/6J background [22]. All animals were housed in the Animal Care Facility of the Toronto General Research Institute with a 12-h light/dark cycle and free access to regular chow diet and water. All procedures were approved by the Animal Care Committee of the University Health Network, University of Toronto.

Study groups Wild-type mice were randomly divided into three experimental groups. The first group, Control mice, were injected i.v. with vehicle of the CB1R antagonist AM251 (saline/DMSO/Tween80, 80:10:10, vol./vol.) $1 \mathrm{~h}$ before start of the euglycaemic-hyperinsulinaemic clamp, and with vehicle of the CB1R agonist HU210 (saline/ ethanol/Tween80, 18:1:1, vol./vol.) $15 \mathrm{~min}$ after initiation of the clamp. The second group, the CB1R agonist group (HU210), were injected i.v. with vehicle of AM251 $1 \mathrm{~h}$ before the start of the clamp and with HU210 (50 ng/g body weight) 15 min after the start of the clamp. The third group, the CB1R antagonist+agonist group (AM251+HU210), were injected i.v. with AM251 (6 $\mu \mathrm{g} / \mathrm{g}$ body weight $) 1 \mathrm{~h}$ preceding start of the clamp, and with HU210 $(50 \mathrm{ng} / \mathrm{g}$ body weight) $15 \mathrm{~min}$ after the start of the clamp. Separate experiments with acute AM251 administration alone showed no effects on clamp variables (electronic supplementary material [ESM] Fig. 1) or insulin-stimulated Akt phosphorylation (ESM Fig. 2). We therefore elected to include only the first of the above groups (Control, vehicle only-treated) as control in further studies. $\mathrm{Cb}_{1 r^{-1}}$ mice were randomly assigned to two experimental groups, which received either HU210 (50 ng/g body weight) or vehicle i.v. $15 \mathrm{~min}$ after the start of the clamp, without pretreatment with antagonist or its vehicle. Two additional groups of wild-type mice received intracerebroventricular (i.c.v.) injection of HU210 (50 ng/g body weight) or vehicle 15 min after the start of clamp studies. AM251 and HU210 were freshly prepared in concentrations according to $2 \mu \mathrm{l} / \mathrm{g}$ body weight injection volume. In preliminary studies, we had determined that $50 \mathrm{ng} / \mathrm{g}$ body weight is an optimal dose to elicit an effect on insulin sensitivity in vivo and is well within the $20-100 \mathrm{ng} / \mathrm{g}$ body weight range commonly used by others $[11,21,23-25]$. The dose of AM251 was the 
same as reported in chronic studies that elicited metabolic effects on feeding and body weight in mice [26-28]. In the i.c.v. experiments, a total volume of $2 \mu \mathrm{H}$ HU210 or vehicle (saline/ethanol/Tween80, 18:1:1 vol./vol.) was injected. This volume has been reported in other studies and shown to be safe in mice [29].

Surgical procedures Mice at 8 to 12 weeks of age were catheterised under general anaesthesia (ketamine $100 \mathrm{mg} /$ $\mathrm{kg}$, xylazine $7.5 \mathrm{mg} / \mathrm{kg}$, i.p.). The right jugular vein was cannulated with a microrenathane catheter (MRE 0.25; Braintree Scientific, Braintree, MA, USA), sealed and tunnelled subcutaneously to the back of the neck as previously described [30]. Mice were allowed 3-5 days to recover. Only mice that recovered uneventfully and lost less than $5 \%$ of their preoperative body weight were used for subsequent experiments.

In two separate groups of wild-type mice, brain infusion cannulae were stereotaxically placed with their tips in the lateral cerebral ventricle using the following coordinates: $0.7 \mathrm{~mm}$ posterior to bregma, $1.0 \mathrm{~mm}$ lateral to the midsagittal suture, both to a depth of $2.2 \mathrm{~mm}$, with bregma and lambda at the same vertical dimension, determined from the mice brain atlas of Franklin and Paxinos [31]. The guide cannulae were secured to the skull surface with two screws and dental cement. After 1 week of recovery, the localisation of the cannulae was confirmed by the instant drinking response to an i.c.v. injection of angiotensin II [32]. The right jugular vein was cannulated as above in mice with successful brain ventricle cannulation. Mice were allowed another 3 to 5 days to recover. Again, only mice that recovered uneventfully were used for subsequent clamp studies.

Euglycaemic-hyperinsulinaemic clamp procedure and determination of tissue glucose uptake Euglycaemichyperinsulinaemic clamps were conducted in fasted, conscious, tail-retained mice as previously described [30] with minor modifications. After baseline blood sampling from the tail vein, a primed $\left(1.85 \times 10^{5} \mathrm{~Bq}\right.$ bolus $)$ constant $(1.85 \times$ $10^{3} \mathrm{~Bq} / \mathrm{min}$ ) infusion of $\left[{ }^{3} \mathrm{H}\right]$ glucose (New England Nuclear, Boston, MA, USA) was initiated and continued for $100 \mathrm{~min}$. Mice were pretreated with AM251 $(6 \mu \mathrm{g} / \mathrm{g}$ body weight) or vehicle $1 \mathrm{~h}$ before the start of the clamp, i.e. $40 \mathrm{~min}$ after start of the basal period. During the last $20 \mathrm{~min}$ of the basal period, blood samples were taken every $10 \mathrm{~min}$ for determination of basal glucose-specific activity, glucose concentrations and plasma hormones. Subsequently, the clamp period was started with infusion of insulin (50 mU/kg body weight bolus $+1.5 \mathrm{mU} \mathrm{kg}^{-1} \mathrm{~min}^{-1}$ infusion) and $\left[{ }^{3} \mathrm{H}\right]$ glucose $\left(1.85 \times 10^{3} \mathrm{~Bq} / \mathrm{min}\right)$. Blood samples were collected every $10 \mathrm{~min}$ and glucose levels measured with a glucometer (Sure Step, One Touch; Lifescan,
Milpitas, CA, USA). A 25\% (wt/vol.) dextrose solution was infused at a variable rate to maintain blood glucose at the basal level. Additional $\left[{ }^{3} \mathrm{H}\right]$ glucose was added to the dextrose infusate to minimise the decline in glucosespecific activity during the clamp. Steady-state of the clamp was achieved between 90 and $120 \mathrm{~min}$, during which time blood samples were collected every $10 \mathrm{~min}$ for determination of glucose-specific activity, glucose concentrations and plasma insulin levels. CB1R agonist HU210 (50 ng/g body weight) or vehicle was administered i.v. or i.c.v. $15 \mathrm{~min}$ after the start of the clamp period. An i.v. bolus of 2-deoxy-D-[1- $\left.{ }^{14} \mathrm{C}\right]$ glucose $(2-\mathrm{DG})\left(3.7 \times 10^{5} \mathrm{~Bq}\right.$; NEN Life Science, Boston, MA, USA) was administered $80 \mathrm{~min}$ before the end of the clamp. At the end of the clamp, mice were killed with sodium pentobarbital. Within $5 \mathrm{~min}$ of death, three pieces of muscle tissue (quadriceps, soleus and gastrocnemius from both hindlimbs) and abdominal visceral adipose tissue were rapidly excised, weighed, immediately frozen in liquid nitrogen and stored at $-70^{\circ} \mathrm{C}$ for later analysis.

Assays Plasma insulin was measured using a radioimmunoassay kit (Millipore, Billerica, MA, USA). For determination of plasma $\left[{ }^{3} \mathrm{H}\right]$ glucose and 2-DG specific activities, plasma was deproteinised with zinc sulphate and barium hydroxide, dried to remove ${ }^{3} \mathrm{H}_{2} \mathrm{O}$ and counted in scintillation fluid on dual channels for separation of ${ }^{3} \mathrm{H}$ and ${ }^{14} \mathrm{C}$ (Beckman Coulter, Fullerton, CA, USA). For determination of tissue 2-DG-6-P content, muscle and adipose tissue samples were homogenised and centrifuged $(1,000 \times g$, $5 \mathrm{~min}$ ), and the supernatant fractions subjected to an ionexchange column to separate 2-DG-6-P from 2-DG, as previously described [33].

Calculations Endogenous glucose production rate (EGP) during clamps was determined by subtracting the glucose infusion rate (Ginf) from whole-body glucose uptake. Insulin-mediated glucose disappearance was the glucose disappearance rate $\left(R_{\mathrm{d}}\right)$ measured with $\left[{ }^{3} \mathrm{H}\right]$ glucose during the clamp minus mean baseline $R_{\mathrm{d}}$ values [34, 35]. Data were smoothed with the optimal segments routine, using the optimal error algorithm [36]. Glucose uptake in individual skeletal muscle and adipose tissues was calculated from plasma 2-DG specific activity and tissue 2-DG6-P content as previously described [37].

Acute in vivo insulin stimulation and analysis ex vivo of skeletal muscle Akt phosphorylation Wild-type mice of 9 weeks of age were fasted overnight before i.v. injection of vehicle, HU210 or AM251+HU210, exactly as in the clamp experiments described above. At the end of the treatments, i.e. time corresponding to the end of the clamp, muscle tissue were collected as described [38]. Briefly, under 
anaesthesia with pentobarbital, the abdominal cavity was opened and portal vein exposed. Gastrocnemius muscle from one hindlimb was rapidly collected and frozen immediately in liquid nitrogen. A maximal bolus of insulin (10 U/kg body weight) was then injected into the portal vein. At $5 \mathrm{~min}$ after insulin injection, gastrocnemius muscle from the opposite hindlimb was collected and immediately frozen. Tissue protein preparation and western blotting were done as previously described [39]. Whole-tissue protein from lysates (approximately $30 \mu \mathrm{g}$ protein) was separated by $10 \%$ SDS-PAGE, transferred to nitrocellulose membranes and incubated with antibodies against Akt, phosphorylated Akt Ser473 and Thr308 (Cell Signaling Technology, Beverly, MA, USA). Detection of blotted proteins was performed by enhanced chemiluminescence (Thermo Fisher Scientific, Rockford, IL, USA) according to the manufacturer's instructions.

Statistical analyses Values are reported as mean \pm SEM. For body weight, glucose, insulin and variables of euglycaemic-hyperinsulinaemic clamps, one-way ANOVA was used to compare the control, CB1R agonist and CB1R antagonist+agonist experimental groups. Differences between the groups were assessed by post-hoc analysis using Tukey's test. A value of $p<0.05$ was considered to be statistically significant.

\section{Results}

General characteristics of animals Body weights, baseline fasting blood glucose and plasma insulin concentrations prior to euglycaemic-hyperinsulinaemic clamp studies were not significantly different between experimental groups for wildtype and $\mathrm{Cblr}^{-/}$mice (Table 1). Fasting insulin levels in $\mathrm{Cb}_{1 r^{-1}}$ mice tended to be lower than in wild-type mice. Although the difference did not reach statistical significance, possibly due to the younger age of mice in our study, the trend towards lower insulin levels in $\mathrm{Cblr}^{--}$than in wildtype mice is consistent with findings of others [16].

Effect of acute CB1R modulation on insulin sensitivity in wild-type and $\mathrm{Cb}^{-1}{ }^{-1}$ mice Glucose and insulin concentrations during the last $30 \mathrm{~min}$ of the clamp were not significantly different between experimental groups for wild-type mice (Table 1). Plasma glucose-specific activity was maintained close to baseline (within $20 \%$ of basal specific activity) and within a similar range during the last $30 \mathrm{~min}$ of the clamp in all experimental groups. In wildtype mice, acute administration of HU210 significantly lowered the Ginf that was required to maintain euglycaemia during the last $30 \mathrm{~min}$ of the clamp $(p<0.01$; Fig. 1a), indicating that $\mathrm{CB} 1 \mathrm{R}$ agonism acutely induced insulin resistance in treated mice. The acute effect of HU210 on induction of insulin resistance was rapid, since the reduction of Ginf occurred as early as $40 \mathrm{~min}$ after clamp initiation. Pretreatment of mice with the selective CB1R antagonist AM251 completely prevented the decrease of Ginf (i.e. the insulin resistance) induced by HU210 in wildtype mice (Fig. 1a). In contrast, HU210 administration had no effect on Ginf (i.e. whole-body insulin sensitivity) in $\mathrm{Cb}^{-/}{ }^{--}$mice (Fig. 2a).

Effect of acute CB1R modulation on EGP and $R_{d}$ in wildtype and $\mathrm{Cblr}^{--}$mice To determine whether the above changes in whole-body insulin sensitivity were due to changes in $R_{\mathrm{d}}$, EGP or both, we calculated these variables by modelling changes in $\left[{ }^{3} \mathrm{H}\right]$ glucose-specific activity as described above. $R_{\mathrm{d}}$ was significantly reduced in wild-type mice treated with HU210 (Fig. 1b), indicating that the CB1R agonist acutely reduced whole-body glucose uptake. Pretreatment with AM251 completely prevented the reduction in $R_{\mathrm{d}}$ induced by HU210 in wild-type mice. However, insulin-induced suppression of EGP during the last $30 \mathrm{~min}$ of the clamp did not differ between experimental groups of wild-type mice (Fig. 1c, d). Antagonist alone did not elicit similar insulin-desensitising effects, as EGP and $R_{\mathrm{d}}$ levels in AM251 group were not statistically significantly different from those in vehicle-treated animals (ESM Fig. 1). In $\mathrm{Cblr}^{-/}$mice, insulin-induced stimulation of $R_{\mathrm{d}}$ and suppression of EGP were not significantly different between HU210- and vehicle-treated groups (Fig. 2b-d).

Effect of acute CB1R modulation on insulin-stimulated glucose uptake in muscle and adipose tissues In wild-type mice, HU210 significantly reduced glucose uptake in soleus (mainly type I fibre), quadriceps (mainly type II fibre) and gastrocnemius (mixture, and type II and type I fibres) muscles, as compared with vehicle-treated group (Table 2). This effect was abolished by pretreatment with AM251. Glucose uptake was similar in adipose tissue in all experimental groups (Table 2).

Effect of acute CBIR modulation in vivo on Akt phosphorylation in muscle tissues To further investigate the mechanisms of CB1R acute modulation of insulin signalling in skeletal muscle, we examined Akt phosphorylation in vivo under acute insulin stimulation after treatment of mice with CB1R agonist and/or antagonist. Insulin-stimulated Akt phosphorylation at Ser473 and Thr308 was significantly impaired by HU210, this impairment being prevented by pre-treatment with AM251 (Fig. 3). AM251 alone did not significantly affect Akt phosphorylation (ESM Fig. 2).

Effect of acute i.c.v. CB1R agonist administration on insulin sensitivity in wild-type mice Ginf (Fig. 4a) during 
Table 1 Body weight, fasting basal and clamped plasma glucose and insulin in wild-type and $\mathrm{Cblr}^{--}$mice

Values are mean \pm SEM

There were no differences in any variables between experimental groups ( $n=6$ in each experimental group)
Variables per mouse type

Experimental groups

\begin{tabular}{lll}
\hline Vehicle & HU210 & AM251+ HU210
\end{tabular}

Wild-type

Body weight (g)

Basal blood glucose $(\mathrm{mmol} / \mathrm{l})$

Clamped blood glucose ( $\mathrm{mmol} / \mathrm{l})$

Basal plasma insulin (pmol/1)

Clamped plasma insulin (pmol/1) $\mathrm{Cb}_{1 r^{-1}}$

Body weight ( $\mathrm{g}$ )

Basal blood glucose (mmol/l)

Clamped blood glucose ( $\mathrm{mmol} / \mathrm{l})$

Basal plasma insulin (pmol/l)

$25.4 \pm 0.6$

$25.2 \pm 0.2$

$24.5 \pm 0.6$

$7.2 \pm 0.3$

$7.0 \pm 0.3$

$6.8 \pm 0.3$

$7.7 \pm 0.3$

$7.3 \pm 0.3$

$7.2 \pm 0.2$

$75.1 \pm 8.6$

$76.6 \pm 5.2$

$81.9 \pm 1.2$

$224.2 \pm 46.7$

$241.7 \pm 13.2$

$277.2 \pm 32.8$

$\begin{array}{ll}23.1 \pm 0.9 & 22.5 \pm 1.1 \\ 6.8 \pm 0.5 & 6.5 \pm 0.8 \\ 7.1 \pm 0.3 & 6.6 \pm 0.4 \\ 62.3 \pm 4.8 & 70.6 \pm 11.7 \\ 201.9 \pm 21.3 & 211 \pm 26.7\end{array}$

a

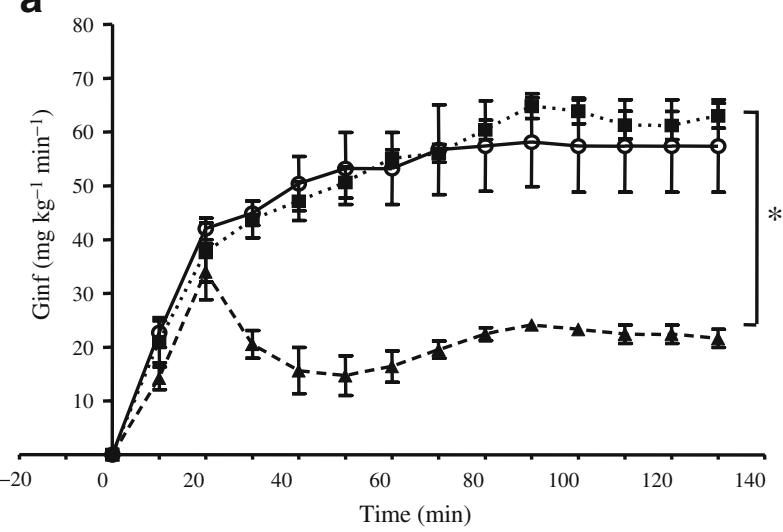

C

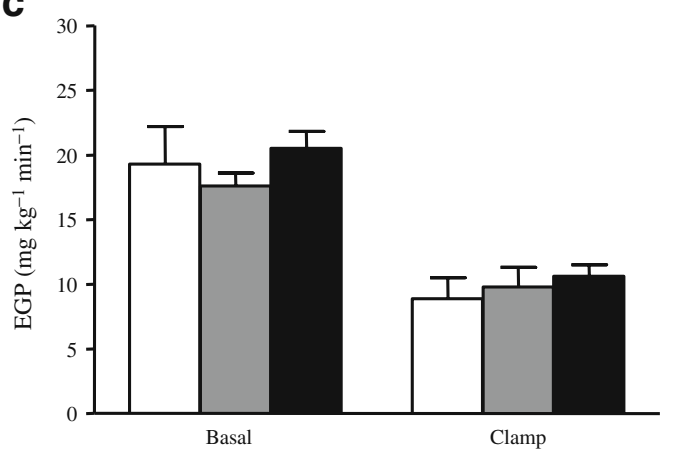

Fig. 1 In wild-type C56BL/6 mice (8-12 weeks of age), CB1R agonist (HU210, $50 \mathrm{ng} / \mathrm{g}$ body weight, i.v., $15 \mathrm{~min}$ after start of clamp at time $0 \mathrm{~min}$ ) (black triangles, grey bars) induced whole-body insulin resistance, as evidenced by a $61 \%$ lower Ginf required to maintain euglycaemia during the last $30 \mathrm{~min}$ of a $120 \mathrm{~min}$ euglycaemichyperinsulinaemic clamp vs vehicle (white circles, white bars). Pretreatment of the mice with a selective CB1R antagonist AM251 (black squares, black bars) $(6 \mu \mathrm{g} / \mathrm{g}$ body weight, i.v., $2 \mathrm{~h}$ before b

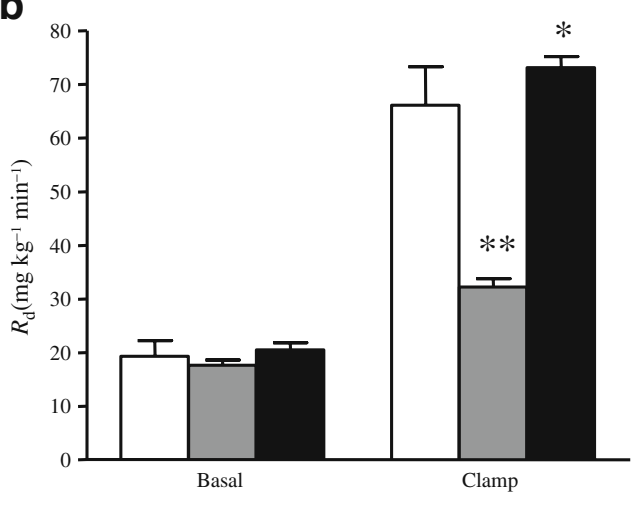

d

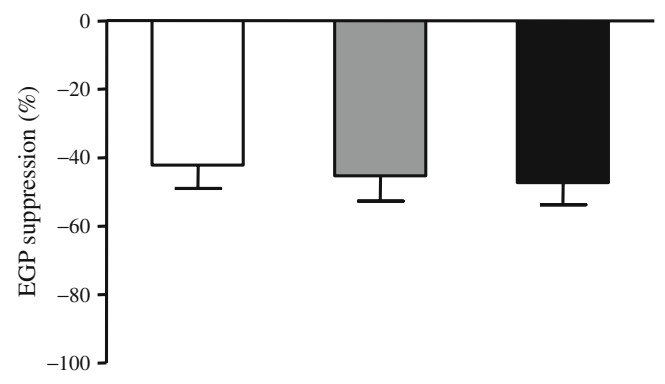

clamp) prevented the development of insulin resistance induced by HU210, as indicated by a similar Ginf to that in vehicle-treated mice (a). The profound impairment of insulin sensitivity induced by HU210 was due to a significant reduction of $R_{\mathrm{d}}(\mathbf{b})$, rather than to diminished insulin suppression of EGP $(\mathbf{c}, \mathbf{d})$. Pretreatment of the mice with AM251 prevented the reduction (b) of $R_{\mathrm{d}}$ induced by HU210. ${ }^{*} p<$ 0.05 vs HU210; $* * p<0.01$ vs vehicle and AM251+HU210 
a

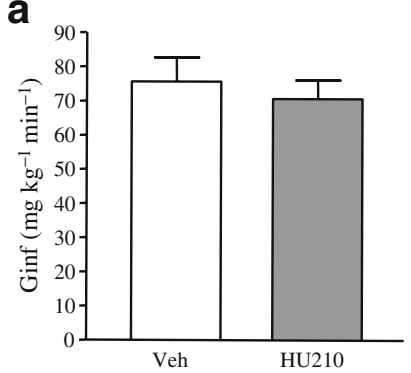

b

C

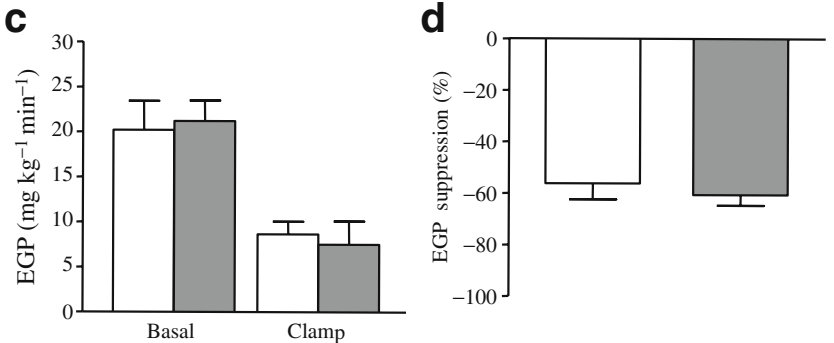

Fig. 2 In $\mathrm{Cblr}^{-/}$mice (8-12 weeks of age), Ginf (a), $R_{\mathrm{d}}$ (b) and EGP (c, d) were not significantly different between HU210-treated group (grey bars) $(50 \mathrm{ng} / \mathrm{g}$ body weight, i.v., $15 \mathrm{~min}$ after start of clamp) and the vehicle-treated group (white bars), indicating resistance of $\mathrm{Cblr}^{-/-}$mice to the acute insulin desensitising effect of HU210; $n=6$

the last 30 min of the clamp were not significantly different between i.c.v. HU210-treated group and i.c.v. vehicletreated group in wild-type mice. EGP and $R_{\mathrm{d}}$ were similar between groups (Fig. 4b-d).

\section{Discussion}

Increasing evidence indicates that the endocannabinoid system is involved in regulation of insulin sensitivity. However, most changes in insulin sensitivity have been shown to accompany weight loss or resistance to dietinduced obesity. For example, plasma insulin levels are low in $\mathrm{Cb}_{1 r^{--}}$mice compared with their wild-type littermates; moreover, the former are resistant to diet-induced obesity and insulin resistance [16]. Plasma insulin levels were reduced in diet-induced obese mice treated with a specific CB1R antagonist SR141716 [13, 40]. In rats after chronic treatment with a CB1R antagonist, insulin sensitivity and skeletal muscle glucose uptake were also enhanced, whereas hepatic glucose production was diminished [20]. Chronic, peripheral CB1R antagonism decreases hepatic glucose production, promotes lipid mobilisation independently of food intake and increases glucose utilisation in rats [20]. Recent in vitro data also suggest that adipocytes may secrete CB1R-activating factors and that CB1Rs in human skeletal muscle participate in the negative crosstalk between fat and muscle [41], with muscle CB1R playing a major role in the development of insulin resistance. $\mathrm{Cblr}^{-/}$ mice do not develop hyperinsulinaemia, hyperleptinaemia and dyslipidaemic profile, even after chronic feeding with high-fat diet $[11,21]$. In clinical studies, insulin sensitivity was significantly improved after 1 or 2 years of treatment with the CB1R antagonist rimonabant [2, 4]. Although these combined data strongly suggest an important role of CB1R in modulating insulin sensitivity and glucose metabolism, it was not possible to separate insulin sensitisation secondary to weight loss from the direct effects of the drug in these studies. Neither the direct role of CB1R in modulating insulin sensitivity in vivo, nor the major tissue sites and mechanisms of action of $\mathrm{CB} 1 \mathrm{R}$ agonists and antagonists in this process are currently well understood. In addition, understanding of CB1R's acute in vivo effects, independent of that on weight loss, in these processes is limited.

To examine the role of $\mathrm{CB} 1 \mathrm{R}$ in modulating insulin sensitivity in vivo, we first assessed the effect of acute gainand loss-of-function of whole-body CB1R on insulin sensitivity using the euglycaemic-hyperinsulinaemic clamp in conscious mice. We showed that, in wild-type mice, administration of the CB1R agonist HU210 acutely induced whole-body insulin resistance, as revealed by the significant $50 \%$ to $60 \%$ reduction of Ginf during the last $30 \mathrm{~min}$ of a 120 min euglycaemic-hyperinsulinaemic clamp. This effect was abolished when the animals were pretreated with a selective CB1R antagonist, AM251. The insulin resistance induced by acute CB1R agonism was accounted for by decreased glucose disposal, which is mainly by skeletal muscle, whereas we did not detect an acute effect on insulin's ability to suppress EGP, which is mainly hepatic (discussed further below). Since EGP was not altered by CB1R agonist HU210, hepatic CB1R does not seem to mediate acute changes in insulin sensitivity. Our findings that the effect of HU210 was completely absent in $\mathrm{Cb}_{1} \mathrm{r}^{-1}$ mice (which lack CB1R in the CNS and outside the CNS), as well as in wild-type mice treated with the CB1R antagonist AM251, prove that it was indeed stimulation of $\mathrm{CB} 1 \mathrm{R}$ that was responsible for the acute reduction in insulin sensitivity, and not an off-target drug effect.

Table 2 2-DG uptake in skeletal muscles and visceral adipose of wild-type mice

\begin{tabular}{llll}
\hline Tissue & Vehicle & HU210 & AM251+HU210 \\
\hline Soleus & $226 \pm 15$ & $75 \pm 17 \dagger$ & $171 \pm 30^{*}$ \\
Quadriceps & $195 \pm 18$ & $68 \pm 8 \dagger$ & $168 \pm 24^{*}$ \\
Gastrocnemius & $202 \pm 21$ & $80 \pm 12 \dagger$ & $185 \pm 14^{*}$ \\
Visceral adipose & $20.6 \pm 5.1$ & $16.1 \pm 3.9$ & $15.7 \pm 5.2$ \\
\hline
\end{tabular}

Values are expressed as mean $\pm \mathrm{SEM}$, with units in $\mu \mathrm{mol} \mathrm{kg} \mathrm{min}^{-1}$ ${ }^{\dagger} p<0.05$ vs Vehicle and AM251+HU210; ${ }^{*} p<0.05$ vs HU210 
a

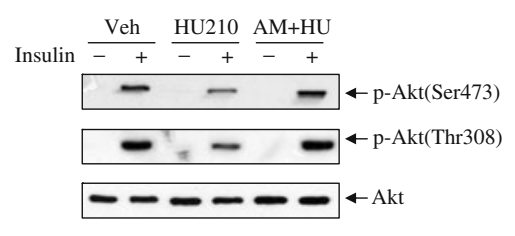

b

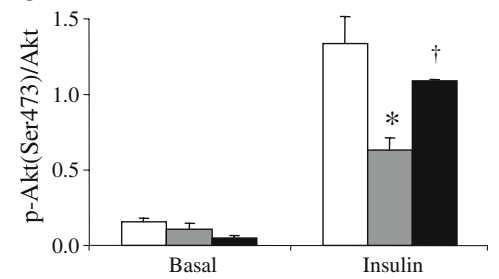

C

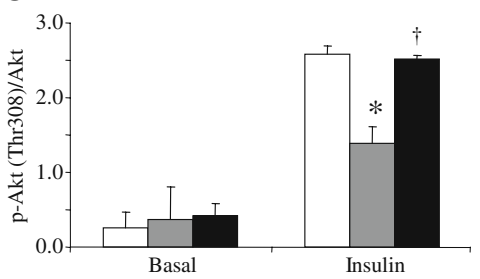

Fig. 3 Insulin-stimulated Akt phosphorylation, as assessed by western blot (a) and quantified for phosphorylated (p)-Akt Ser473 (b) and Thr308 (c), in muscle of wild-type mice was reduced by acute i.v. injection of CB1R agonist HU210 (50 ng/g body weight, $105 \mathrm{~min}$ before muscle collection) (grey bars) compared with vehicle-treated animals (white bars), which was prevented by pre-treatment with CB1R antagonist AM251 $(6 \mu \mathrm{g} / \mathrm{g}$ body weight, $180 \mathrm{~min}$ before muscle collection) (black bars). ${ }^{*} p<0.05$ vs vehicle (Veh); ${ }^{\dagger} p<0.05$ vs HU210

2-DG uptake in adipose tissue in these experiments, indicating that adipose tissue CB1R plays a negligible role in mediating the acute changes of insulin sensitivity with CB1R agonism/antagonism. Altered glucose and lipid metabolism, and insulin sensitivity have been shown with chronic modulation of CB1R in peripheral tissues, including the liver $[18,20]$. Our study is complementary to the anticipated long-term effects of CB1R modulation on insulin sensitivity, in that we demonstrate an acute prominent effect on insulin action in muscle.

Although our results indicate that peripheral tissues such as skeletal muscle are major tissue sites responsible for the change in insulin sensitivity with acute CB1R modulation, the change of $R_{\mathrm{d}}$ induced by HU210 in wild-type mice could still have been secondary to CNS regulation of CB1R activity. Over the past decade, there has been mounting evidence that activation of the neural circuits in the hypothalamus can control hepatic and extra-hepatic carbohydrate metabolism [42]. To rule out this possibility, we administered HU210 or vehicle into the right brain ventricle in a separate group of wild-type mice during a euglycaemichyperinsulinaemic clamp. We found that i.c.v. injection of HU210 did not induce insulin resistance as seen when the same dose of HU210 was administered peripherally in wildtype mice. We deliberately chose to administer the same dose of HU210 i.c.v. as that we had administered systemically to demonstrate an acute insulin desensitising effect of HU210, reasoning that the i.c.v. CNS drug concentration with this dose would exceed the CNS concentration achieved with systemic administration of the drug. It is possible that this relatively high dose administered i.c.v. could have leaked out of the CNS into the systemic circulation, thereby exerting systemic effects on insulin sensitivity. However, the total absence of effect on insulin sensitivity with i.c.v. administration rules out this possibility in this experiment. These findings suggest that CB1Rs in the brain are not involved in the acute change of insulin sensitivity induced by systemic HU210 administration. Thus the insulin-desensitising effect of CB1R agonism in peripheral tissues are not likely to have
Fig. 4 In wild-type C56BL/6 mice (8-12 weeks of age), Ginf (a), $R_{\mathrm{d}}$ (b) and EGP (c, d) were not significantly different between HU210treated group (grey bars) (i.c.v., $50 \mathrm{ng} / \mathrm{g}$ body weight, $15 \mathrm{~min}$ after start of clamp) and the vehicle treated group (white bars) $(n=6)$, indicating that i.c.v. administration of HU210 failed to reproduce the acute insulin desensitisation that was observed when the identical dose was administered systemically (i.v.) 
been mediated by CNS factors in our experiments. Our findings are consistent with accumulating evidence suggesting that part of the metabolic effect of chronic CB1R antagonists is independent of reduction in energy intake, pointing to a role for peripheral tissue CB1R in induction of weight loss. In humans [43] and animal models [10, 12-16], changes in body weight during CB1R agonist or antagonist treatments can be independent of food intake, suggesting that CB1R plays a role in directly mediating metabolism. In accordance with our finding that $\mathrm{CB} 1 \mathrm{R}$ mediates muscle insulin sensitivity, the role of CB1R in mediating energy expenditure has previously been shown to occur in peripheral tissues, by which mechanism a non-brain-penetrant CB1R antagonist reduced body weight and improved metabolism in mice [44].

The potential mechanisms involved in changes in insulin sensitivity following CB1R modulation remain unclear. It has been shown in vitro that insulin signalling may be affected by short- to long-term modulation of CB1R activity in muscle cells through differing mechanisms [41, 45]. In L6 cells, $24 \mathrm{~h}$ activation of CB1R with $\mathrm{N}$-(2chloroethyl)-5Z,8Z,11Z,14Z-eicosatetraenamide (ACEA) decreased signalling mediated by extracellular signalregulated protein kinase (ERK)1/2, but not signalling mediated by Akt, while antagonism with SR141716 increased Akt and ERK1/2 activation [45]. Our finding that acute agonism of CB1R impaired insulin-stimulated Akt phosphorylation is in agreement with several in vitro studies that have shown rapid effects of CB1R modulation on insulin signalling. For example, Esposito et al. demonstrated that SR141716 stimulated 2-DG uptake in L6 cells through phosphatidylinositol 3-kinase and downstream proteins, including Akt, an effect observed as early as $30 \mathrm{~min}$ post treatment [46]. In human skeletal muscle cells, treatment with the CB1R agonist anandamide for $1 \mathrm{~h}$ increased ERK1/ 2 and p38 mitogen-activated protein kinase, and impaired insulin-stimulated phosphorylation of Akt (Ser473), but not of Akt (Thr308) [41]. These studies indicate that tonic modulation of CB1R can lead to changes in insulin action in muscle, possibly through the cross-talk between endocannabinoid system and insulin signalling pathways. On the other hand, it is possible that the effects on muscle insulin sensitivity are secondary to systemic factors in the in vivo setting. For instance, CB1R modulation affects adipose tissue production and secretion of adiponectin [10, 47], which is closely associated with insulin sensitivity [48]. Although changes in insulin sensitivity by CB1R modulation may be linked to adiponectin in chronic conditions [49, 50], acute modulation of CB1R is not expected to significantly modify adiponectin production and secretion.

We conclude that extrahepatic insulin sensitivity is impaired after acute stimulation of non-CNS CB1R, most likely of CB1Rs residing in skeletal muscle. Our studies did not address the clinical question of whether treatment of insulin-resistant humans with a CB1R antagonist exerts its beneficial metabolic effect via CB1Rs outside the CNR. However, we speculate from our findings of acute modulation of CB1R that this may indeed be an important mechanism of action of this promising class of therapeutic agents. Our findings support the development and application of non-brain-penetrant CB1R antagonists that improve insulin sensitivity and ameliorate metabolic abnormalities accompanying insulin-resistant states.

Acknowledgements We would like to thank G. Kunos (National Institutes of Health) for providing us with $\mathrm{Cb} 1 \mathrm{r}$ knockout mice and T. Lam for help with the i.c.v. techniques. D. Song is the recipient of a postdoctoral research award from the Banting and Best Diabetes Centre, University of Toronto. G. F. Lewis holds a Canada Research Chair in Diabetes and the Drucker Family Chair in Diabetes Research, and is a Career Investigator of the Heart and Stroke Foundation of Canada.

Duality of interest The authors declare that there is no duality of interest associated with this manuscript.

\section{References}

1. Aranceta J, Moreno B, Moya M, Anadon A (2009) Prevention of overweight and obesity from a public health perspective. Nutr Rev 67:S83-S88

2. Despres JP, Golay A, Sjostrom L (2005) Effects of rimonabant on metabolic risk factors in overweight patients with dyslipidemia. $\mathrm{N}$ Engl J Med 353:2121-2134

3. Di Marzo V, Despres JP (2009) CB1 antagonists for obesitywhat lessons have we learned from rimonabant? Nat Rev Endocrinol 5:633-638

4. Pi-Sunyer FX, Aronne LJ, Heshmati HM, Devin J, Rosenstock J (2006) Effect of rimonabant, a cannabinoid-1 receptor blocker, on weight and cardiometabolic risk factors in overweight or obese patients: RIO-North America: a randomized controlled trial. JAMA 295:761-775

5. Matias I, Di MV (2007) Endocannabinoids and the control of energy balance. Trends Endocrinol Metab 18:27-37

6. Pagotto U, Marsicano G, Cota D, Lutz B, Pasquali R (2006) The emerging role of the endocannabinoid system in endocrine regulation and energy balance. Endocr Rev 27:73-100

7. Bensaid M, Gary-Bobo M, Esclangon A et al (2003) The cannabinoid CB1 receptor antagonist SR141716 increases Acrp30 mRNA expression in adipose tissue of obese fa/fa rats and in cultured adipocyte cells. Mol Pharmacol 63:908-914

8. Cavuoto P, McAinch AJ, Hatzinikolas G, Cameron-Smith D, Wittert GA (2007) Effects of cannabinoid receptors on skeletal muscle oxidative pathways. Mol Cell Endocrinol 267:63-69

9. Cavuoto P, McAinch AJ, Hatzinikolas G, Janovska A, Game P, Wittert GA (2007) The expression of receptors for endocannabinoids in human and rodent skeletal muscle. Biochem Biophys Res Commun 364:105-110

10. Cota D, Marsicano G, Tschop M et al (2003) The endogenous cannabinoid system affects energy balance via central orexigenic drive and peripheral lipogenesis. J Clin Invest 112:423-431

11. Osei-Hyiaman D, DePetrillo M, Pacher P et al (2005) Endocannabinoid activation at hepatic $\mathrm{CB} 1$ receptors stimulates fatty acid synthesis and contributes to diet-induced obesity. J Clin Invest 115:1298-1305 
12. Herling AW, Gossel M, Haschke G et al (2007) CB1 receptor antagonist AVE1625 affects primarily metabolic parameters independently of reduced food intake in Wistar rats. Am J Physiol Endocrinol Metab 293:E826-E832

13. Ravinet TC, Arnone M, Delgorge C et al (2003) Anti-obesity effect of SR141716, a CB1 receptor antagonist, in diet-induced obese mice. Am J Physiol Regul Integr Comp Physiol 284:R345-R353

14. Herling AW, Kilp S, Elvert R, Haschke G, Kramer W (2008) Increased energy expenditure contributes more to the body weight-reducing effect of rimonabant than reduced food intake in candy-fed Wistar rats. Endocrinology 149:2557-2566

15. Cota D, Sandoval DA, Olivieri M et al (2009) Food intakeindependent effects of CB1 antagonism on glucose and lipid metabolism. Obesity (Silver Spring) 17:1641-1645

16. Ravinet TC, Delgorge C, Menet C, Arnone M, Soubrie P (2004) CB1 cannabinoid receptor knockout in mice leads to leanness, resistance to diet-induced obesity and enhanced leptin sensitivity. Int J Obes 28:640-648

17. Herling AW, Kilp S, Juretschke HP, Neumann-Haefelin C, Gerl M, Kramer W (2008) Reversal of visceral adiposity in candy-diet fed female Wistar rats by the CB1 receptor antagonist rimonabant. Int J Obes (Lond) 32:1363-1372

18. Tam J, Vemuri VK, Liu J et al (2010) Peripheral CB1 cannabinoid receptor blockade improves cardiometabolic risk in mouse models of obesity. J Clin Invest 120:2953-2966

19. Kunos G (2007) Understanding metabolic homeostasis and imbalance: what is the role of the endocannabinoid system? Am J Med 120:S18-S24

20. Nogueiras R, Veyrat-Durebex C, Suchanek PM et al (2008) Peripheral, but not central, CB1 antagonism provides food intake independent metabolic benefits in diet-induced obese rats. Diabetes 57:2977-2991

21. Osei-Hyiaman D, Liu J, Zhou L et al (2008) Hepatic CB1 receptor is required for development of diet-induced steatosis, dyslipidemia, and insulin and leptin resistance in mice. J Clin Invest 118:3160-3169

22. Zimmer A, Zimmer AM, Hohmann AG, Herkenham M, Bonner TI (1999) Increased mortality, hypoactivity, and hypoalgesia in cannabinoid CB1 receptor knockout mice. Proc Natl Acad Sci USA 96:5780-5785

23. Dalton VS, Wang H, Zavitsanou K (2009) HU210-induced downregulation in cannabinoid $\mathrm{CB} 1$ receptor binding strongly correlates with body weight loss in the adult rat. Neurochem Res 34:1343-1353

24. Giuliani D, Ottani A, Ferrari F (2000) Effects of the cannabinoid receptor agonist, HU 210, on ingestive behaviour and body weight of rats. Eur J Pharmacol 391:275-279

25. Robinson L, Goonawardena AV, Pertwee RG, Hampson RE, Riedel G (2007) The synthetic cannabinoid HU210 induces spatial memory deficits and suppresses hippocampal firing rate in rats. $\mathrm{Br}$ J Pharmacol 151:688-700

26. Chen RZ, Frassetto A, Fong TM (2006) Effects of the CB1 cannabinoid receptor inverse agonist AM251 on food intake and body weight in mice lacking $\mu$-opioid receptors. Brain Res 1108:176-178

27. Irwin N, Hunter K, Frizzell N, Flatt PR (2008) Antidiabetic effects of sub-chronic administration of the cannabinoid receptor $(\mathrm{CB}(1))$ antagonist, AM251, in obese diabetic (ob/ob) mice. Eur J Pharmacol 581:226-233

28. Irwin N, Hunter K, Flatt PR (2008) Comparison of independent and combined chronic metabolic effects of GIP and CB1 receptor blockade in high-fat fed mice. Peptides 29:1036-1041

29. Mistry AM, Swick AG, Romsos DR (1997) Leptin rapidly lowers food intake and elevates metabolic rates in lean and ob/ob mice. J Nutr 127:2065-2072

30. Kim JK, Gavrilova O, Chen Y, Reitman ML, Shulman GI (2000) Mechanism of insulin resistance in A-ZIP/F-1 fatless mice. J Biol Chem 275:8456-8460
31. Franklin F, Paxinos G (1998) The mouse brain in stereotaxic coordinates. Academic Press, Sydney

32. Osborn JL, Camara AK (1997) Renal neurogenic mediation of intracerebroventricular angiotensin II hypertension in rats raised on high sodium chloride diet. Hypertension 30:331-336

33. Ohshima K, Shargill NS, Chan TM, Bray GA (1984) Adrenalectomy reverses insulin resistance in muscle from obese (ob/ob) mice. Am J Physiol 246:E193-E197

34. Ayala JE, Bracy DP, McGuinness OP, Wasserman DH (2006) Considerations in the design of hyperinsulinemic-euglycemic clamps in the conscious mouse. Diabetes 55:390-397

35. Wang $\mathrm{CH}$, Leung $\mathrm{N}$, Lapointe $\mathrm{N}$ et al (2003) Vasopeptidase inhibitor omapatrilat induces profound insulin sensitization and increases myocardial glucose uptake in Zucker fatty rats: studies comparing a vasopeptidase inhibitor, angiotensin-converting enzyme inhibitor, and angiotensin II type I receptor blocker. Circulation 107:1923-1929

36. Bradley DC, Steil GM, Bergman RN (1993) Quantitation of measurement error with optimal segments: basis for adaptive time course smoothing. Am J Physiol 264:E902-E911

37. Youn JH, Kim JK, Buchanan TA (1994) Time courses of changes in hepatic and skeletal muscle insulin action and GLUT4 protein in skeletal muscle after STZ injection. Diabetes 43:564-571

38. Shao J, Yamashita H, Qiao L, Friedman JE (2000) Decreased Akt kinase activity and insulin resistance in C57BL/KsJ-Leprdb/db mice. J Endocrinol 167:107-115

39. Shao W, Yu Z, Fantus IG, Jin T (2010) Cyclic AMP signaling stimulates proteasome degradation of thioredoxin interacting protein (TxNIP) in pancreatic beta-cells. Cell Signal 22:1240-1246

40. Jourdan T, Djaouti L, Demizieux L, Gresti J, Verges B, Degrace P (2010) CB1 antagonism exerts specific molecular effects on visceral and subcutaneous fat and reverses liver steatosis in dietinduced obese mice. Diabetes 59:926-934

41. Eckardt K, Sell H, Taube A et al (2009) Cannabinoid type 1 receptors in human skeletal muscle cells participate in the negative crosstalk between fat and muscle. Diabetologia 52:664-674

42. Lam CK, Chari M, Lam TK (2009) CNS regulation of glucose homeostasis. Physiology (Bethesda) 24:159-170

43. Greenberg I, Kuehnle J, Mendelson JH, Bernstein JG (1976) Effects of marihuana use on body weight and caloric intake in humans. Psychopharmacology (Berl) 49:79-84

44. LoVerme J, Duranti A, Tontini A et al (2009) Synthesis and characterization of a peripherally restricted CB1 cannabinoid antagonist, URB447, that reduces feeding and body-weight gain in mice. Bioorg Med Chem Lett 19:639-643

45. Lipina C, Stretton C, Hastings S et al (2010) Regulation of MAP kinase-directed mitogenic and protein kinase B-mediated signaling by cannabinoid receptor type 1 in skeletal muscle cells. Diabetes 59:375-385

46. Esposito I, Proto MC, Gazzerro P et al (2008) The cannabinoid CB1 receptor antagonist rimonabant stimulates 2-deoxyglucose uptake in skeletal muscle cells by regulating the expression of phosphatidylinositol-3-kinase. Mol Pharmacol 74:1678-1686

47. Poirier B, Bidouard JP, Cadrouvele C et al (2005) The anti-obesity effect of rimonabant is associated with an improved serum lipid profile. Diabetes Obes Metab 7:65-72

48. Civitarese AE, Ukropcova B, Carling S et al (2006) Role of adiponectin in human skeletal muscle bioenergetics. Cell Metab 4:75-87

49. Engeli S, Heusser K, Janke J et al (2008) Peripheral endocannabinoid system activity in patients treated with sibutramine. Obesity Silver Spring 16:1135-1137

50. Migrenne S, Lacombe A, Lefevre AL et al (2009) Adiponectin is required to mediate rimonabant-induced improvement of insulin sensitivity but not body weight loss in diet-induced obese mice. Am J Physiol Regul Integr Comp Physiol 296:R929-R935 